\title{
PERIODIC ORBITS OF THE TWO FIXED CENTERS PROBLEM WITH A VARIATIONAL GRAVITATIONAL FIELD
}

\author{
FABAO GAO ${ }^{1,2}$, JAUME LLIBRE ${ }^{2}$
}

\begin{abstract}
Within a given range of energy levels the two fixed centers problem under a variational gravitational field admits periodic orbits bifurcating from the Kepler problem. The analytical expressions of these periodic orbits are given when the mass parameter of the system is sufficiently small.
\end{abstract}

\section{IntRoduction AND STATEMENT OF THE MAIN RESUlt}

It is well known that due to the non-integratable feature of the restricted three-body problem. Scientists have not yet obtained analytical expressions of its general solutions, and that its periodic orbits have extremely important applications in practical space missions. This fact which has attracted a large number of mathematicians and astronomers to carry out research on the periodic orbits of the restricted three-body problem (see [1] and the references therein). The extensive research covered three categories: qualitative analysis (see [1]-[4] and so on), analytical calculation (see [5]-[7] ), and numerical simulation (see [8][13]).

For the case of the planar circular restricted three-body problem, Zotos [14] investigated the problem with two equivalent masses with strong gravitational field, which was controlled by power of the gravitational potential. They revealed the influences of the gravitational potential power on the nature of orbits. Based on the continuation method, Llibre and Paşca [15] proved that the circular and the elliptic symmetric periodic orbits of the planar rotating Kepler problem can be continued into periodic orbits of the planar collision restricted threebody problem. The method was also applied by Llibre and Makhlouf

Key words and phrases. three-body problem, periodic orbit, averaging theory, variational gravitational field. 
[16] to provide sufficient conditions of periodic solutions of a fourthorder differential system. For large value of eccentricity $e$, Abouelmagd et al. [17] found that the anisotropic Kepler problem with small anisotropy has two periodic orbits in every negative energy level bifurcating from elliptic orbits of the Kepler problem by using averaging theory. In addition, they also presented the approximate analytic expressions of the continued periodic orbits. Recently, for a sufficiently small parameter and each value of eccentricity $e \in(0,1)$, Llibre and Yuan [18] continued elliptic periodic orbits of the Kepler problem to the the planar anisotropic Manev problem and of two perturbations of the hydrogen atom problem.

As for the analytical calculation of periodic orbits of three-body problem, there were already scientists conducting research on it as early as a few decades ago. In 1973, Farquhar and Kamel [5] developed an approximation for periodic orbit, spurring a spate of investigation into the computation of periodic orbits. Richardson [6], [7] constructed the classical periodic 'halo' orbits seven years later, he presented a third-order analytical solution for halo-type periodic motion about the collinear points of the circular restricted problem based on the method of successive approximations and a technique similar to the LindstedtPoincaré method.

Mainly motivated by Zotos [14] and Llibre [16] , this paper focuses on the question of what kind of periodic orbits of the Kepler problem can be continued to two fixed centers with the a variational gravitational field, and presenting the analytical expression of periodic orbits.

1.1. Equations of Motion. The equations of motion of a particle in the two fixed centers problem with a variational gravitational field can be written as

$$
q_{i}^{\prime}=\frac{\partial H}{\partial p_{i}}, \quad p_{i}^{\prime}=-\frac{\partial H}{\partial q_{i}}, \quad \text { for } i=1,2,
$$

where

$$
H=\frac{1}{2}\left(p_{1}^{2}+p_{2}^{2}\right)-\frac{1-\mu}{r_{1}}-\frac{\mu}{r_{2}^{p}},
$$

and $r_{1}=\sqrt{\left(q_{1}+\mu\right)^{2}+q_{2}^{2}}, r_{2}=\sqrt{\left(q_{1}-1+\mu\right)^{2}+q_{2}^{2}}$.

Here the prime represents derivative with respect to the time $t$ and $\mu$ $(0<\mu \ll 1)$ denotes the mass parameter of the two masses, one of mass $1-\mu$ fixed at $(-\mu, 0)$ and the other of mass $\mu$ fixed at $(1-\mu, 0)$. The parameter $p$ represents the power of the gravitational potential. When 
$p$ is negative, then the interaction is variationaler for short distances and stronger for large distances. Zotos in [14] studied the restricted circular three-body problem when $p>1$. Here we shall study the two fixed centers problem with the $p=-2$.

Our main result is the following:

Theorem 1. For the mass parameter $\mu>0$ sufficiently small and for every value $h \in(-1 / 2,0)$, the elliptic periodic solution with eccentricity $e=-2 h$ of the Kepler problem can be continued to the two fixed centers problem with variational gravitational field $p=-2$.

\section{Proof of THEOREM}

Introducing the McGehee coordinates $(r, \theta, v, u)$, of which $r$ and $\theta$ are the radius and the angle in polar coordinate, $v$ and $u$ are the scaled radial and angular components of velocity, respectively, as follows

$$
\begin{aligned}
\left(q_{1}, q_{2}\right) & =r(\cos \theta, \sin \theta), \\
r^{-1 / 2} v & =\left(p_{1}, p_{2}\right) \cdot(\cos \theta, \sin \theta), \\
r^{-1 / 2} u & =\left(p_{1}, p_{2}\right) \cdot(-\sin \theta, \cos \theta) .
\end{aligned}
$$

Then the Hamiltonian of system (2) with $p=-2$ becomes

$$
\begin{aligned}
H= & \frac{1}{2}\left(p_{1}^{2}+p_{2}^{2}\right)-\frac{1}{\sqrt{q_{1}^{2}+q_{2}^{2}}} \\
& +\mu\left(-1+2 q_{1}-q_{1}^{2}-q_{2}^{2}+\frac{q_{1}+q_{1}^{2}+q_{2}^{2}}{\left(q_{1}^{2}+q_{2}^{2}\right)^{3 / 2}}\right)+\mathcal{O}\left(\mu^{2}\right),
\end{aligned}
$$

and we have

$$
\begin{aligned}
r^{\prime}= & r^{-1 / 2} v \\
\theta^{\prime}= & r^{-3 / 2} u \\
v^{\prime}= & r^{-3 / 2}\left(u^{2}+\frac{v^{2}}{2}-1\right) \\
& +\mu r^{-5 / 2}\left[r\left(1+2 r^{3}\right)+2\left(1-r^{3}\right) \cos \theta\right]+\mathcal{O}\left(\mu^{2}\right) \\
u^{\prime}= & r^{-3 / 2}\left(-\frac{1}{2} u v\right)+\mu r^{-5 / 2}\left(1+2 r^{3}\right) \sin \theta+\mathcal{O}\left(\mu^{2}\right) .
\end{aligned}
$$


Note that $r=0$ corresponds to the collision singularity of equations (4). Let $\mathrm{d} t / \mathrm{d} \tau=r^{5 / 2}$, hence equations (4) can be rewritten as

$$
\begin{aligned}
\dot{r}= & r^{2} v, \\
\dot{\theta}= & r u, \\
\dot{v}= & r\left(u^{2}+\frac{v^{2}}{2}-1\right) \\
& +\mu\left[r\left(1+2 r^{3}\right)+2\left(1-r^{3}\right) \cos \theta\right]+\mathcal{O}\left(\mu^{2}\right), \\
\dot{u}= & r\left(-\frac{1}{2} u v\right)+\mu\left(1+2 r^{3}\right) \sin \theta+\mathcal{O}\left(\mu^{2}\right),
\end{aligned}
$$

where the dot denotes derivative with respect to the new time $\tau$.

Taking into account that the Hamiltonian system (3) is reduced to the Kepler problem when $\mu=0$, we will study what periodic orbits of the Kepler problem can be continued to a given negative energy level of the two fixed centers problem with variational gravitational field $p=-2$.

For a given energy level $H=h$ we have

$$
\frac{1}{2}\left(u^{2}+v^{2}\right)-1+\mu\left[1-r-r^{3}+\frac{\left(1+2 r^{3}\right)}{r} \cos \theta\right]=r h .
$$

Isolating $r=r(\theta, v, u, h)$ from equation (6) in power series of the small mass parameter $\mu$ we obtain

$$
r=r(\theta, v, u, h)=R_{0}+\mu R_{1}+\mathcal{O}\left(\mu^{2}\right),
$$

where

$$
\begin{aligned}
R_{0}= & \frac{1}{2 h}\left(u^{2}+v^{2}-2\right), \\
R_{1}= & \frac{1}{8 h^{4}}\left[8 h^{3}-4 h^{2}\left(u^{2}+v^{2}-2\right)-\left(u^{2}+v^{2}-2\right)^{3}\right] \\
& +\frac{1}{2 h^{3}} \frac{4 h^{3}+\left(u^{2}+v^{2}-2\right)^{3}}{u^{2}+v^{2}-2} .
\end{aligned}
$$


Therefore substituting equation (7) into equations (5) and taking $\theta$ as the new independent variable, equations (5) become

$$
\begin{aligned}
& \frac{\mathrm{d} v}{\mathrm{~d} \theta}=\frac{2 u^{2}+v^{2}-2}{2 u}+\mu\left[\frac{1}{u}+\frac{\left(u^{2}+v^{2}-2\right)^{3}}{4 h^{3} u}\right. \\
&\left.+\frac{8 h^{3}-\left(u^{2}+v^{2}-2\right)^{3}}{2 h^{2}\left(u^{2}+v^{2}-2\right) u} \cos \theta\right]+\mathcal{O}\left(\mu^{2}\right), \\
& \frac{\mathrm{d} u}{\mathrm{~d} \theta}=-\frac{v}{2}+\mu \frac{4 h^{3}+\left(u^{2}+v^{2}-2\right)^{3}}{2 h^{2}\left(u^{2}+v^{2}-2\right) u} \sin \theta+\mathcal{O}\left(\mu^{2}\right) .
\end{aligned}
$$

If $\mu=0$ equations (8) will be reduced to the following the unperturbed system

$$
\frac{\mathrm{d} v}{\mathrm{~d} \theta}=\frac{2 u^{2}+v^{2}-2}{2 u}
$$

$$
\frac{\mathrm{d} u}{\mathrm{~d} \theta}=-\frac{v}{2}
$$

which admits the general solution

$$
\begin{aligned}
& v\left(\theta ; e, \theta_{0}\right)=\frac{e \sin \left(\theta-\theta_{0}\right)}{\sqrt{1+e \cos \left(\theta-\theta_{0}\right)}}, \\
& u\left(\theta ; e, \theta_{0}\right)=\sqrt{1+e \cos \left(\theta-\theta_{0}\right)}
\end{aligned}
$$

where $\theta_{0} \in[0,2 \pi)$ and $e$ are the argument of pericenter and the eccentricity of the planar Kepler problem, respectively. The eccentricity $e=0$ corresponds to circular periodic solutions, and $e \in(0,1)$ indicates that the corresponding periodic solutions are elliptical ones.

In order to apply the averaging theory described in the Appendix to equations (8), we do the following transformations

$$
\begin{aligned}
& \mathbf{x}=\left(\begin{array}{l}
v \\
u
\end{array}\right), \mathbf{x}(\theta ; \mathbf{z}, 0)=\left(\begin{array}{c}
v\left(\theta ; e, \theta_{0}\right) \\
u\left(\theta ; e, \theta_{0}\right)
\end{array}\right), \mathbf{z}=\left(\begin{array}{c}
e \\
\theta_{0}
\end{array}\right), \\
& \mathbf{F}_{\mathbf{0}}=\left(\begin{array}{l}
F_{01} \\
F_{02}
\end{array}\right), \mathbf{F}_{\mathbf{1}}=\left(\begin{array}{c}
F_{11} \\
F_{12}
\end{array}\right),
\end{aligned}
$$


where

$$
\begin{aligned}
& F_{01}=\frac{2 u^{2}+v^{2}-2}{2 u}, \\
& F_{02}=-\frac{v}{2}, \\
& F_{11}=\frac{1}{u}+\frac{\left(u^{2}+v^{2}-2\right)^{3}}{4 h^{3} u}+\frac{8 h^{3}-\left(u^{2}+v^{2}-2\right)^{3}}{2 h^{2}\left(u^{2}+v^{2}-2\right) u} \cos \theta, \\
& F_{12}=\frac{4 h^{3}+\left(u^{2}+v^{2}-2\right)^{3}}{2 h^{2}\left(u^{2}+v^{2}-2\right) u} \sin \theta .
\end{aligned}
$$

Now we calculate the averaged function $\mathcal{F}(\mathbf{z})$ in equation (15) in the Appendix.

Let

$$
\left(G_{1}\left(\theta ; e, \theta_{0}\right), G_{2}\left(\theta ; e, \theta_{0}\right)\right)=M_{\mathbf{z}}^{-1}(\theta, \mathbf{z}) \mathbf{F}_{\mathbf{1}}(\theta, \mathbf{x}(\theta ; \mathbf{z}, 0))
$$

where

$$
M_{\mathbf{z}}^{-1}(\theta, \mathbf{z})=\left(\begin{array}{ll}
y_{1}\left(\theta ; e, \theta_{0}\right) & y_{2}\left(\theta ; e, \theta_{0}\right) \\
y_{3}\left(\theta ; e, \theta_{0}\right) & y_{4}\left(\theta ; e, \theta_{0}\right)
\end{array}\right)
$$

denotes the inverse of the fundamental matrix of equations (9). Here

$$
\begin{aligned}
y_{1}\left(\theta ; e, \theta_{0}\right)= & \frac{\left(1+e \cos \left(\theta-\theta_{0}\right)\right)^{1 / 2}}{2\left(1+e \cos \theta_{0}\right)^{3 / 2}}\left[2 \cos \theta\left(1+e \cos \theta_{0}\right)\right. \\
& \left.+e \sin \theta \sin \theta_{0}\right], \\
y_{2}\left(\theta ; e, \theta_{0}\right)=- & \frac{1}{8\left(1+e \cos \left(\theta-\theta_{0}\right)\right)^{1 / 2}\left(1+e \cos \theta_{0}\right)^{3 / 2}} \\
\cdot & {\left[2\left(8+e^{2} \cos \theta+8 e \cos \cos \theta_{0}\right) \sin \theta\right.} \\
& +2\left(4 \cos \theta_{0}+3 e \cos 2 \theta_{0}\right) \sin 2 \theta \\
& +16 e(2+\cos \theta) \sin ^{2}(\theta / 2) \sin \theta_{0} \\
& \left.+6 e^{2} \sin ^{2} \theta \sin 2 \theta_{0}\right] \\
\left(1+e \cos \left(\theta-\theta_{0}\right)\right)^{1 / 2} & \sin \theta \\
y_{3}\left(\theta ; e, \theta_{0}\right)= & \frac{\left(1+e \cos \theta_{0}\right)^{1 / 2}}{2\left(1 \cos \theta+e\left(\cos \left(2 \theta-\theta_{0}\right)+3 \cos \theta_{0}\right)\right.} \\
y_{4}\left(\theta ; e, \theta_{0}\right)= & \frac{4\left(1+e \cos \left(\theta-\theta_{0}\right)\right)^{1 / 2}\left(1+e \cos \theta_{0}\right)^{1 / 2}}{4(1+2}
\end{aligned}
$$


Hence

$$
\begin{aligned}
& G_{1}\left(\theta ; e, \theta_{0}\right) \\
& =\frac{1}{8\left(1+e \cos \theta_{0}\right)^{3 / 2}}\left[\left(4+\frac{\left(-1+e^{2}\right)^{3}}{h^{3}\left(1+e \cos \left(\theta-\theta_{0}\right)\right)^{3}}\right.\right. \\
& +\frac{16 h \cos \theta\left(1+e \cos \left(\theta-\theta_{0}\right)\right)}{-1+e^{2}} \\
& \left.\cdot\left(1-\frac{\left(-1+e^{2}\right)^{3}}{8 h^{3}\left(1+e \cos \left(\theta-\theta_{0}\right)\right)^{3}}\right)\right) \\
& \text { - }\left(2 \cos \theta\left(1+e \cos \theta_{0}\right)+e \sin \theta \sin \theta_{0}\right) \\
& +\frac{2 h \sin \theta}{-1+e^{2}}\left(1+\frac{\left(-1+e^{2}\right)^{3}}{4 h^{3}\left(1+e \cos \left(\theta-\theta_{0}\right)\right)^{3}}\right) \\
& \cdot\left(-2\left(8+e^{2} \cos \theta+8 e \cos \theta_{0}\right) \sin \theta\right. \\
& -e\left(4 \cos \theta_{0}+3 e \cos 2 \theta_{0}\right) \sin 2 \theta \\
& \left.\left.-16 e(2+\cos \theta) \sin \left(\frac{\theta}{2}\right)^{2} \sin \theta_{0}-6 e^{2} \sin ^{2} \theta \sin 2 \theta_{0}\right)\right] \text {, } \\
& G_{2}\left(\theta ; e, \theta_{0}\right) \\
& =\frac{\sin \theta}{8\left(1+e \cos \theta_{0}\right)^{1 / 2}}\left[\left(4+\frac{\left(-1+e^{2}\right)^{3}}{h^{3}\left(1+e \cos \left(\theta-\theta_{0}\right)\right)^{3}}\right.\right. \\
& +\frac{16 h \cos \theta\left(1+e \cos \left(\theta-\theta_{0}\right)\right)}{-1+e^{2}} \\
& \left.\cdot\left(1-\frac{\left(-1+e^{2}\right)^{3}}{8 h^{3}\left(1+e \cos \left(\theta-\theta_{0}\right)\right)^{3}}\right)\right) \\
& +\frac{4 h\left(4 \cos \theta+e\left(\cos \left(2 \theta-\theta_{0}\right)+3 \cos \theta_{0}\right)\right)}{-1+e^{2}} \\
& \left.\cdot\left(1+\frac{\left(-1+e^{2}\right)^{3}}{4 h^{3}\left(1+e \cos \left(\theta-\theta_{0}\right)\right)^{3}}\right)\right] .
\end{aligned}
$$

Substituting equations (11) into equation (15) in the Appendix and computing the corresponding integrals we obtain

$$
\mathcal{F}(\mathbf{z})=\left(f_{1}\left(e, \theta_{0}\right), f_{2}\left(e, \theta_{0}\right)\right),
$$


where

$$
\begin{aligned}
f_{1}\left(e, \theta_{0}\right)= & \frac{3 \sqrt{1-e^{2}}}{32 h^{3}\left(1+e \cos \theta_{0}\right)^{3 / 2}}\left[3 e^{2}-8 h\right. \\
& \left.-4 e(-1+2 h) \cos \theta_{0}+e^{2} \cos 2 \theta_{0}\right] \\
f_{2}\left(e, \theta_{0}\right)= & \frac{3 e \sqrt{1-e^{2}} \sin \theta_{0}}{16 h^{3} \sqrt{1+e \cos \theta_{0}}} .
\end{aligned}
$$

The function $\mathcal{F}(\mathbf{z})$ in equation (12) is the averaged function of system (8). According with the averaging theory (see the Appendix) we must compute the simple zeros of the system $f_{1}\left(e, \theta_{0}\right)=0, f_{2}\left(e, \theta_{0}\right)=0$. Here we must consider $e \neq 0$, otherwise $f_{2}\left(e, \theta_{0}\right) \equiv 0$, and then the averaging theory does not provide any information. From the equation $f_{2}\left(e, \theta_{0}\right)=0$ we get

$$
\theta_{0}^{1}=0, \theta_{0}^{2}=\pi
$$

Case I: If $\theta_{0}^{1}=0$, we have $e=1$ or $e=2 h$ from the first equation of (13). However, both values of $e$ should be discarded because $e \in(0,1)$ and $h<0$.

Case II: If $\theta_{0}^{2}=\pi$, we have $e=1$ or $e=-2 h$ from the first equation of (13), and $e=1$ should also be discarded because it does not provide a periodic solution of the Kepler problem. Thus $e=-2 h$ with the $h \in(-1 / 2,0)$.

Note that the Jacobian

$$
J=\left|\begin{array}{ll}
\frac{\partial g_{1}}{\partial e} & \frac{\partial g_{1}}{\partial \theta_{0}} \\
\frac{\partial g_{2}}{\partial e} & \frac{\partial g_{2}}{\partial \theta_{0}}
\end{array}\right|_{\left(e, \theta_{0}\right)=(-2 h, \pi)}=\frac{9(2 h-1)}{64 h^{5}} \neq 0 .
$$

Therefore if $h \in(-1 / 2,0)$, we find that the periodic solution

$$
(v(\theta ;-2 h, \pi), u(\theta ;-2 h, \pi))=\left(\frac{2 h \sin \theta}{\sqrt{1+2 h \cos \theta}}, \sqrt{1+2 h \cos \theta}\right)
$$

can be continued to a periodic solution of the two fixed center problem with variational gravitational field $p=-2$ when the mass parameter $\mu$ is sufficiently small. This completes the proof of Theorem 1. 


\section{APPENDix}

We consider the problem of the bifurcation of $T$-periodic solutions from the following system

$$
\dot{\mathbf{x}}=\mathbf{F}_{\mathbf{0}}(t, \mathbf{x})+\mu \mathbf{F}_{\mathbf{1}}(t, \mathbf{x})+\mu^{2} \mathbf{F}_{\mathbf{2}}(t, \mathbf{x}, \mu),
$$

with $\mu=0$ to $\mu \neq 0$ sufficiently small. Here $\mathbf{F}_{\mathbf{0}}, \mathbf{F}_{\mathbf{1}}: \mathbb{R} \times \Omega \rightarrow \mathbb{R}^{n}$, $\mathbf{F}_{2}: \mathbb{R} \times \Omega \times\left(-\mu_{0}, \mu_{0}\right) \rightarrow \mathbb{R}^{n}$ are $\mathcal{C}^{2}$ functions and $T$-periodic with respect to variable $t$, and $\Omega$ is an open subset of $\mathbb{R}^{n}$.

We assume that there is a submanifold $V$ of periodic solutions with the same period of the unperturbed system $\dot{\mathbf{x}}=\mathbf{F}_{\mathbf{0}}(t, \mathbf{x})$. Let $\mathbf{x}(t, \mathbf{z}, \mu)$ be the solution of this unperturbed system such that $\mathbf{x}(0, \mathbf{z}, \mu)=\mathbf{z}$. Then its linearization system along a periodic $\mathbf{x}(t, \mathbf{z}, 0)$ can be written as $\dot{\mathbf{y}}=D_{\mathbf{x}} \mathbf{F}_{\mathbf{0}}(t, \mathbf{x}(t, \mathbf{z}, 0)) \mathbf{y}$, and the fundamental matrix can be denoted by $M_{\mathbf{z}}(t)$.

Supposing that there exists an open set $V$ with $C l(V) \subset \Omega$ such that $\mathbf{x}(t, \mathbf{z}, 0)$ is $T$-periodic for each $\mathbf{z} \in C l(V)$ and $\mathbf{x}(0, \mathbf{z}, 0))=\mathbf{z}$. Here $C l(V)$ denotes the closure of $V$ in $\mathbb{R}^{n}$. Then we have the following result (please see corollary 1 of [19] for an easy proof):

If there is an open and bounded set $V$ with $C l(V) \subset \Omega$ such that $\mathbf{x}(t, \mathbf{z}, 0)$ is T-periodic for each $\mathbf{z} \in C l(V)$. Consider the function $\mathbf{F}: C l(V) \rightarrow \mathbb{R}^{n}$, i.e.,

$$
\mathcal{F}(\mathbf{z})=\frac{1}{T} \int_{0}^{T} M_{\mathbf{z}}^{-1}(\theta, \mathbf{z}) \mathbf{F}_{\mathbf{1}}(\theta, \mathbf{x}(\theta ; \mathbf{z}, 0)) \mathrm{d} \theta .
$$

Assume that there exists $a \in V$ such that $\mathcal{F}(\mathbf{a})=\mathbf{0}$ and $\operatorname{det}((\mathrm{d} \mathcal{F} / \mathrm{d} \mathbf{x})(a)) \neq$ 0 , then there is a T-periodic solution $\phi(t, \mu)$ of system (14) such that $\phi(0, \mu) \rightarrow$ a as $\mu \rightarrow 0$.

\section{ACKNOWLEDGMENTS}

The first author gratefully acknowledges the support of the National Natural Science Foundation of China (NSFC) through grant Nos.11672259 and 11571301, the China Scholarship Council through grant No.201908320086, the Ministry of Land and Resources Research of China in the Public Interest through grant No.201411007.

The second author gratefully acknowledges the support of the Ministerio de Economía, Industria y Competitividad, Agencia Estatal de Investigación grants MTM2016-77278-P (FEDER) and MDM-20140445, the Agència de Gestió d'Ajuts Universitaris i de Recerca grant 
2017SGR1617, and the H2020 European Research Council grant MSCARISE-2017-777911.

\section{REFERENCES}

[1] Musielak Z.E., Quarles B., The three-body problem, Reports on Progress in Physics 77: 065901, 2014.

[2] Gómez G., Ollé M., Second-species solutions in the circular and elliptic restricted three-body problem I. existence and asymptotic approximation, $\mathrm{Ce}$ lestial Mechanics and Dynamical Astronomy 52: 107-146, 1991.

[3] Koon W.S., Lo M.W., Marsden J.E., Ross S.D., Dynamical systems, the threebody problem and space mission design, Marsden Books, ISBN 978-0-61524095-4, 2011.

[4] Gao F.B., Zhang W., A study on periodic solutions for the circular restricted three-body problem, The Astronomical Journal 148: 116, 2014.

[5] Farquhar R.W., Kamel A.A., Quasi-periodic orbits about the translunar libration point, Celestial Mechanics 7: 458-473, 1973.

[6] Richardson D.L., Halo orbit formulation for the ISEE-3 mission, Journal of Guidance and Control 3: 543-548, 1980.

[7] Richardson D.L., Analytical construction of periodic orbits about the collinear points, Celestial Mechanics 22: 241-253, 1980.

[8] Hénon M., Generating families in the restricted three-body problem, SpringerVerlag, 1997.

[9] Moore C., Braids in classical dynamics, Physical Review Letters 70: 3675-3679, 1993.

[10] Chenciner A., Montgomery R., A remarkable periodic solution of the threebody problem in the case of equal masses, Annals of Mathematics 152: 881901, 2000.

[11] Simó C., Dynamical properties of the figure eight solution of the three-body problem, "Celestial Mechanics, dedicated to Donald Saari for his 60th Birthday", A. Chenciner, R. Cushman, C. Robinson, Z.J. Xia ed., Contemporary Mathematics 292: 209-228, 2002.

[12] Šuvakov M., Dmitrašinović V., Three classes of Newtonian three-body planar periodic orbits, Physical Review Letters 110: 114301, 2013.

[13] Li, X.M., Jing, Y.P. and Liao, S.J., Over a thousand new periodic orbits of a planar three-body system with unequal masses, Publications of the Astronomical Society of Japan 70: 64, 2018.

[14] Zotos E.E., Investigating the planar circular restricted three-body problem with strong gravitational field, Meccanica 52: 1995-2021, 2017.

[15] Llibre J., Paşca D., Periodic orbits of the planar collision restricted 3-body problem, Celestial Mechanics and Dynamical Astronomy 96: 19-29, 2006.

[16] Llibre J., Makhlouf A., On the limit cycles for a class of fourth-order differential equations, Journal of Physics. A: Mathematical and Theoretical 45: 055214, 2012.

[17] Abouelmagd E.I., Llibre J., García Guirao J.L., Periodic orbits of the planar anisotropic Kepler problem, International Journal of Bifurcation and Chaos 27: $1750039,2017$. 
[18] Llibre J., Yuan P.F., Periodic orbits of the planar anisotropic Manev problem and of the perturbed hydrogen atom problem, Qualitative Theory of Dynamical Systems: 1-18, 2019.

[19] Buică A., Françoise J.P., Llibre J., Periodic solutions of nonlinear periodic differential systems with a small parameter, Communications on Pure \& Applied Analysis 6: 103-111, 2007.

${ }^{1}$ School of Mathematical Science, Yangzhou University, Yangzhou 225002, CHINA

E-mail: gaofabao@sina.com (Fabao Gao)

2 Departament de Matemàtiques, Universitat Autònoma de Barcelona, Bellaterra 08193, Barcelona, Catalonia, Spain

E-mail: jllibre@mat.uab.cat (Jaume Llibre) 